\title{
CResearch Square
}

\section{A Nonreciprocal Angular Selective Absorber with the Special Multilayer Structure}

\section{Baofei Wan}

Nanjing University of Posts and Telecommunications

\section{Yu Ma}

Nanjing University of Posts and Telecommunications

\section{Ziwei Zhou}

Nanjing University of Posts and Telecommunications

\section{Dan Zhang}

Nanjing Forestry University

Hai-Feng Zhang ( $\nabla$ hanlor@163.com )

Nanjing University of Posts and Telecommunications

\section{Research Article}

Keywords: Angular selective absorber, Nonreciprocal performance, Indium tin oxide film, Transfer matrix method

Posted Date: July 7th, 2021

DOl: https://doi.org/10.21203/rs.3.rs-605098/v1

License: (c) (i) This work is licensed under a Creative Commons Attribution 4.0 International License. Read Full License

Version of Record: A version of this preprint was published at Optical and Quantum Electronics on January 4th, 2022. See the published version at https://doi.org/10.1007/s11082-021-03465-y. 


\title{
A nonreciprocal angular selective absorber with the special multilayer
}

\section{structure}

\author{
Baofei Wan', Yu Ma1, Ziwei Zhou', Dan Zhang² and Haifeng Zhang ${ }^{1-3, \text { a) }}$ \\ ${ }^{1}$ College of Electronic and Optical Engineering \& College of Microelectronics, Nanjing University of Posts and Telecommunications, \\ Nanjing 210023, China \\ ${ }^{2}$ College of Information Science and Technology, Nanjing Forestry University, Nanjing 210037, China \\ ${ }^{3}$ State Key Laboratory of Millimeter Waves of Southeast University, Nanjing 210096, China
}

\begin{abstract}
Due to the orderly design of the special anti-reflection structure and the absorption structure, the one-dimensional layered periodic structure has a good impedance matching in a certain frequency band and a large-angle range, providing for the realization of an angular selective absorber (ASA) with a high rectangle coefficient. For the sake of obtaining excellent absorptivity, the indium tin oxide (ITO) film is used, and it also acts as a function of tuning the absorption angle range (AAR) of the ASA by adjusting the plasma frequency. The proportional relationship between the thickness of the dielectric layers is also discussed to satisfy a good absorption function. At the same time, the ASA also possesses productive nonreciprocal performance (NP) and can also be controlled by the plasma frequency. The transfer matrix method is used for numerical simulation. Our special tunable ASA with the NP is relatively rare in previous studies, which can be applied to optical communications and military fields. Furthermore, we hope that the design we proposed can provide new possibilities for the development of the ASAs.
\end{abstract}

Keyword: Angular selective absorber; Nonreciprocal performance; Indium tin oxide film; Transfer matrix method

\section{Introduction}

The one-dimensional layered periodic structure [1-2] has been widely used in the past decade due to its flexibility in manufacturing, small size, and strong functionality. It plays an indispensable role in the fields of sensors [3-5], filters [6-8], and absorbers [9-11]. Nowadays, angular selective function devices (ASFDs) [12-15] have been sought after by researchers owing to their common application scenarios in the military and communications fields. In Ref.[16], an angular selective filter based on the Brewster angle mode is presented. Attributed to the inherent limitation of the Brewster angle, this ASFD can only be applied in the TM mode with very small angle selectivity. In Ref.[17], an ASFD based on the generalized Brewster mode has attracted people's attention. The researchers have realized a wide range of angle selection functions by cascading Brewster angles, about $20^{\circ}$. Their structures enlarge the angle range, but they cannot solve the problem affected by the polarization state of electromagnetic (EM) waves. In Ref.[13], a wide-angle ASFD that is not affected by the polarization mode is studied. The structure they proposed shows properties independent of the polarization state in the dispersion model, so the defect layer can make part of the light propagate at a specific frequency in the photonic band gap like

\footnotetext{
*Corresponding author at: College of Electronic and Optical Engineering \& College of Microelectronics,Nanjing University of Posts and Telecommunications, Nanjing, 210023 ,China

E-mail: hanlor@163.com ( Hai-Feng Zhang)
} 
a Fabry-Perot resonance. Unfortunately, their proposed filtering structure is not tunable. Benefiting from the efforts of a large number of predecessors, the development of the ASFDs has gradually become fuller. However, previous studies on the ASFDs with productive performance are more about the implementation of filter pieces, and few people can realize the angular selective absorber (ASA) with a high absorption rate and high rectangular coefficient. In addition, the tunability and non-reciprocity of this kind of absorber based on angle selection are also studied.

Optical nonreciprocal devices play a very important role in optical communications [18-20], such as photodiodes and optical isolators. A common mechanism for achieving nonreciprocal performance (NP) is to excite the nonreciprocal surface waves. However, the excitation of such surfaces is difficult after all and needs to meet specific excitation conditions. The structure we proposed can avoid this shortcoming through the clever design of the structure. Indium tin oxide (ITO) films are popular with various absorber researches on account of its tunability and excellent absorption performance [21-23]. Fortunately, the realization of the ASA put forward in this article also profits from it.

In this research, a tunable ASA with interesting NP is raised. Based on the premise of large impedance matching, we can view from the dispersion relationship graph that the incident EM waves can be passed through at a certain frequency and within a certain angle range, possessing very good angle selection characteristics. The transfer matrix method is used to explore the effects of different thicknesses of the dielectric layers, the ratio between the thickness of different dielectric layers, and the plasma frequency on the generation and performance of the ASA. It is worth mentioning that through the ingenious design of the structure, the NP can also be acquired, and the tunability can be completed by adjusting the plasma frequency. We hope that these very fascinating phenomena will promote the development of the optical communications and military fields to a certain extent.

\section{Structure design and simulation}

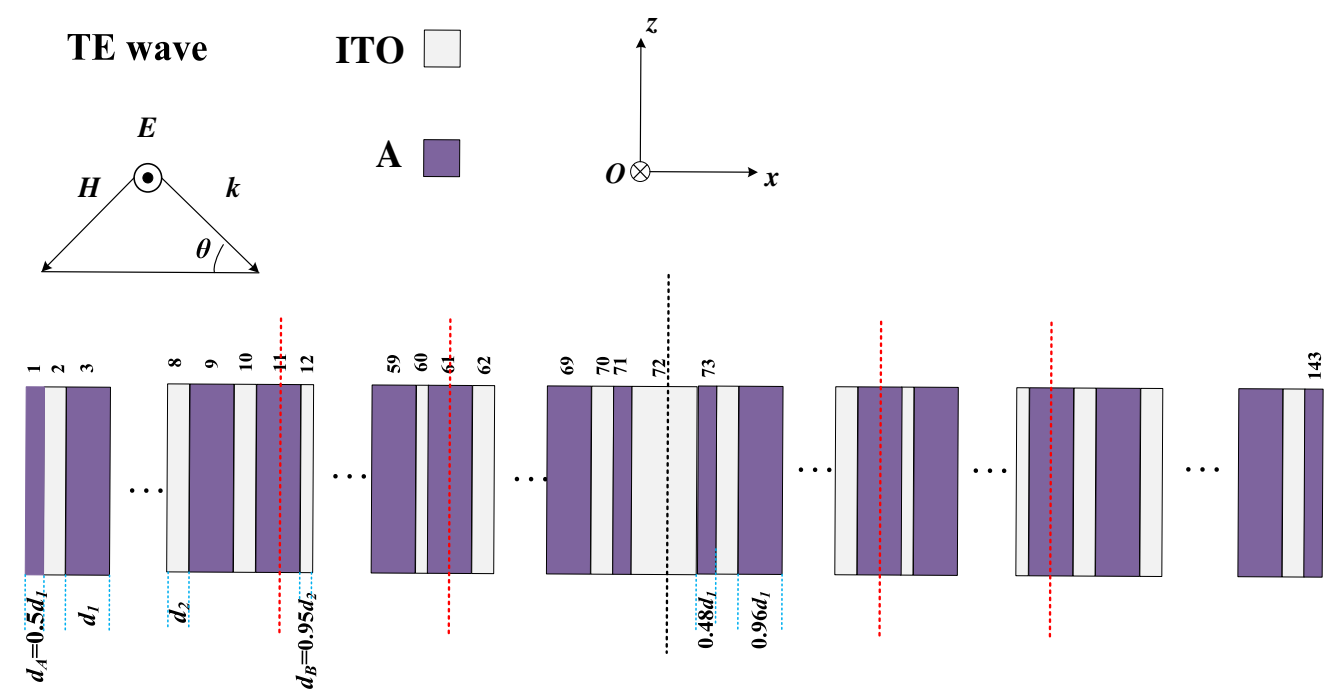

Fig.1. The proposed structure diagram.

As displayed in Fig.1, the model consists of the A dielectric layers and ITO films alternately forming a one-dimensional layered structure. To better describe the model, each layer is marked with a number. The first layer where the incident light enters is named 1 , and the last layer where the incident light exits is named 143 . The entire structure is symmetrical with the 72nd layer as the center in the arrangement of the dielectric layers. In the upper 
half of the black dotted line (in the 72nd layer), the thickness of A of the first and 71st layers is $d_{A}=0.5 d_{l}$, and the thickness of the remaining medium A layers is $d_{l}=225 \mathrm{~nm}$. Between the second to the tenth layers and between the $62 \mathrm{nd}$ to the 70th layers, the thickness of the ITO layer is $d_{2}=85 \mathrm{~nm}$, and that of the remaining ITO layers is $d_{B}=0.95 d_{2}$. The thickness of $72 \mathrm{nd}$ layer of ITO is $2000 \mathrm{~nm}$. In the lower half of the black dashed line, that is, layer 143 to layer 73. The thickness of A at the symmetrical position with the upper half of the black dotted line becomes $0.48 d_{l}$ and $0.96 d_{l}$, respectively, but the thickness of the ITO layer at the symmetrical position does not change.The refractive index of $\mathrm{A}$ is $n_{A}=2.2$. The dielectric function of the ITO film can be calculated using a simplified Drewder model inferred from Ref.[24]:

$$
\varepsilon=\varepsilon_{\infty}-\frac{\omega_{p}^{2}}{\omega(\omega+i \gamma)} .
$$

Where, $\varepsilon_{\infty}=3.9$ means the high-frequency dielectric constant. $\omega_{p}=\left(e^{2} n_{e} / \varepsilon_{0} / m_{e f f}\right)^{1 / 2}$ is the plasma frequency, $n_{e}$ means the plasma density. $\varepsilon_{0}$ represents the vacuum dielectric constant and $e$ is the electronic power. $m_{e f f}=0.35 m$ and $m$ are relatively the effective mass of electrons and electronic quality. $\omega=2 \pi f$ stands for the angular frequency of the incident wave and $f$ means the frequency. $\gamma$ is the plasma collision frequency. The $\omega_{p}$ of the ITO of the upper half of the black dashed line, the lower half of the black dashed line, and the 72nd layer are individually $\omega_{p l}=3880$ $\mathrm{THz}, \omega_{p 2}=3880 \mathrm{THz}$, and $\omega_{p 3}=5000 \mathrm{THz}$. The corresponding plasma collision frequencies are $\gamma_{1}=0.0001 \omega_{p 1}$, $\gamma_{2}=0.0001 \omega_{p 2}$, and $\gamma_{3}=0.1 \omega_{p 3}$. The incident EM wave is the TE wave. $\theta$ refers to the angle formed by the incident light and the $+x$ direction. A clockwise direction is a positive number, and a counterclockwise direction is a negative number.

The propagation of the EM waves in the medium is expressed by the total transfer matrix as [25]:

$$
M=\left[\begin{array}{ll}
M_{11} & M_{12} \\
M_{21} & M_{22}
\end{array}\right]
$$

The reflection and transmission coefficients are calculated as follows [24]:

$$
\begin{aligned}
& r=\frac{\left(M_{11}+M_{12} \eta_{0}\right) \eta_{0}-\left(M_{21}+M_{22} \eta_{0}\right)}{\left(M_{11}+M_{12} \eta_{0}\right) \eta_{0}+\left(M_{21}+M_{22} \eta_{0}\right)}, \\
& t=\frac{2 \eta_{0}}{\left(M_{11}+M_{12} \eta_{0}\right) \eta_{0}+\left(M_{21}+M_{22} \eta_{0}\right)} .
\end{aligned}
$$

In which, $\eta_{0}=\left(\varepsilon_{0} / \mu_{0}\right)^{1 / 2} / \cos \theta$, therefore, the corresponding reflectance and transmittance are:

$$
\begin{aligned}
& R=|r|^{2}, \\
& T=|t|^{2} .
\end{aligned}
$$


Hence, we can get its absorptance $A=1-R-T$.

This paper aims at the theoretical design of a new type of absorber with a high rectangular angle selection function and focuses more on the pursuit of theoretical optimization results. The research focus is not on the specific manufacturing, but it can be manufactured with reference to the scheme in Ref.[26].

\section{Analysis and discussion}

In Fig.2, apparently, in the frequency band higher than $514 \mathrm{THz}$, the absorption effect is excellent (red area in the figure), and has a very good rectangular coefficient (the red area has smooth edges). However, when the frequency is lower than $514 \mathrm{THz}$, the incident light will be exposed irregularly at large angles, seriously affecting the performance of the absorber, which is undesirable. To observe the absorption performance of the ASA more clearly, we define a variable $B$ to represent the rectangular coefficient. $B=G / H$, where, $G$ is the angular selective width when the absorptance is $-30 \mathrm{~dB}$, while $H$ is that in the case of the absorptance is $-3 \mathrm{~dB}$ [27]. The highest and lowest values of $B$ are 1 and 0 . The larger the value of $B$, the higher the steepness and the better the absorption property. If the frequency band satisfying the condition that the absorptance is higher than 0.9 and $B$ is over 0.95 is selected as the effective area, then the ASA we put forward can work in the range of $514 \mathrm{THz} 548 \mathrm{THz}$, such a large bandwidth can meet certain requirements in practical applications. Considering the convenience of the explanation, $f=530 \mathrm{THz}$ is chosen as the research object.

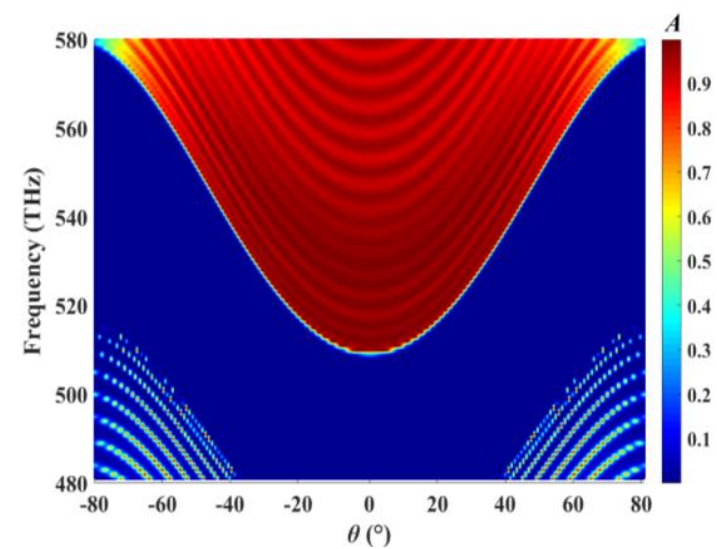

Fig.2. A top view of the absorptance affected $\theta$.

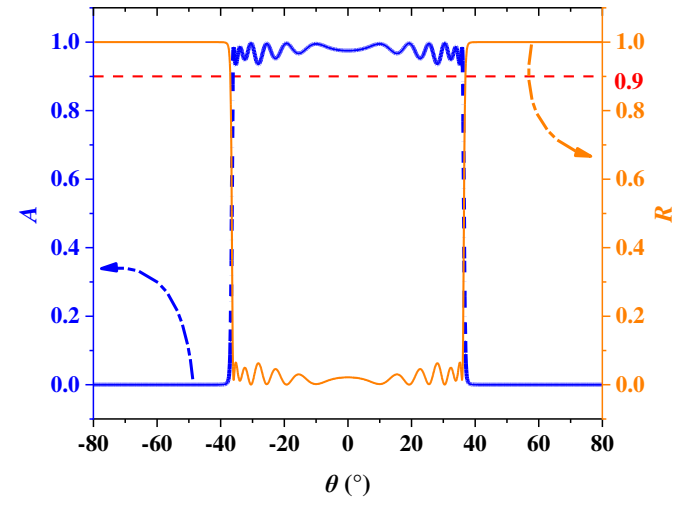

Fig.3. $f=530 \mathrm{THz}$, the spectrum of reflectance $R$ and absorptance $A$ with respect to the change of $\theta$.

As exhibited in Fig.3, suppose $f=530 \mathrm{THz}$, the energy of the incident light is mainly absorbed or reflected, which is the effective mechanism for satisfying the ASA. Precisely, within the absorption angle range (AAR) is $-36.5^{\circ} \sim 36.5^{\circ}$, the absorptance exceeds 0.9 , and $B$ is close to 1 , which is almost a vertical drop, greatly compensating for the traditional ASAs' low rectangularity. These favourable performances are mainly due to the artful design of the structure. On account that the structure shown in Fig.1 is structurally symmetrical about the 72nd floor, whose ultra-high plasma frequency and ultra-wide thickness prevent the incident wave from entering the rear structure, for both forward and backward incidences, the effects produced by the structures on both sides are also similar and independent, so we only take the upper half of the black dotted line for an explanation. The first to the 11th layers and the 61st to the 71th layers are actually two anti-reflection structures, so that part of the EM waves can be propagated in the structure. The 72nd layer serves as an absorber. With the help of its larger thickness 
and higher $\omega_{p 3}$, it produces strong absorption. Coupled with the superposition of more periodic structures from the 11 st layer to the 61 st layer, a high $B$ value will be well guaranteed.

For the sake of expounding the mechanism of the ASA more distinctly, we combine the dispersion theory and impedance matching theory to analyze the structure. From the perspective of impedance matching, the reason for the appearance of the ASA is the good impedance matching of the entire structure and free space. The normalized surface impedance is defined as the ratio of the impedance of the entire structure to the impedance of the vacuum wave [28]:

$$
\frac{Z_{e f f}}{Z_{0}}=z_{r, e f f}+j z_{i, e f f}=\frac{1+r}{1-r}
$$

In which, $Z_{0}=\left|E_{0}\right| /\left|H_{0}\right|=\left(\mu_{0} / \varepsilon_{0}\right)^{1 / 2}$ is the vacuum wave impedance, whose value is about $377 \Omega$. $Z_{\text {eff }}=|E| \Lambda H \mid=(\mu / \varepsilon)^{1 / 2}$ is the effective impedance of the entire structure. $r$ infers the reflection coefficient. In order to fully suppress reflection, the designed structure needs to achieve impedance matching. In general, it is believed that the inhibition is relatively sufficient when the reflectivity is less than 0.1 , and the reflection coefficient is less than 0.32 , in which case, the normalized surface impedance is in the range of $0.51-1.94$. That is to say, when the normalized surface impedance is between 0.51 and 1.94 , the structure involved can fully suppress the reflection and the impedance matching is good. In Fig.4, within the range of $-36.5^{\circ} \sim 36.5^{\circ}$, the real part of the normalized surface impedance is around 0.9 , which belongs to the range of 0.51-1.94, indicating good impedance matching and fully suppressing reflection, providing conditions for the production of high absorption.

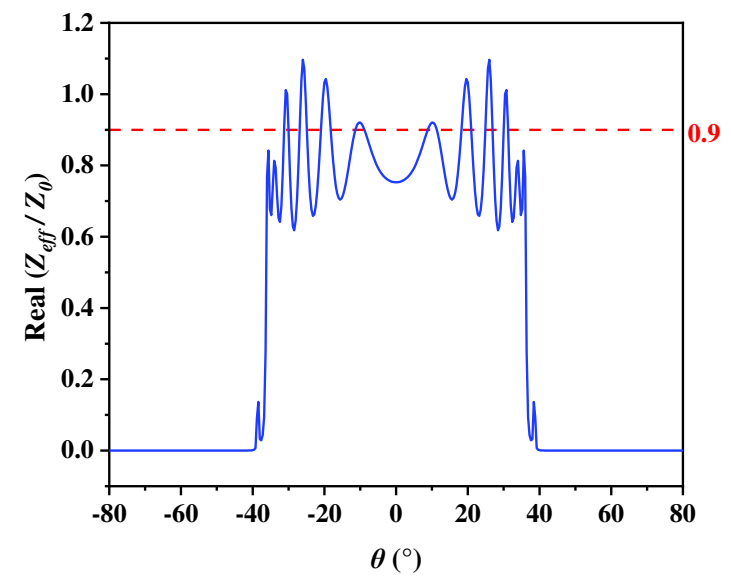

Fig.4. The relationship between the real part of the normalized surface impedance and $\theta$.

Fig.5 is taken to explain from the perspective of dispersion. Suppose $\theta$ changes, only when the frequency does not fall within the photonic forbidden band, can it be propagated. From the perspective of the spectrum, when $k d / 2 \pi$ ( $k$ represents wave vector and $d=d_{l}+d_{B}$ ) is not equal to 0, that is, when it falls in the red area in the picture, the EM waves can be propagated.

In Fig.6, if $d_{l}$ is set as $220 \mathrm{~nm}, 225 \mathrm{~nm}$, or $230 \mathrm{~nm}$, the corresponding AAR values are individual $-28.4^{\circ} \sim 28.4^{\circ}$, $-36.5^{\circ} \sim 36.5^{\circ}$, and $-43.6^{\circ} \sim 43.6^{\circ}$. With the expansion of $d_{l}$, the AAR also tends to increase, broadening by $16.2^{\circ}$ and $14.2^{\circ}$ in sequence. At the same time, the absorptance of the ASA has maintained a level of more than 0.9 , and $B$ is also surpassing 0.95 . Visibly, an appropriate amplification in $d_{l}$ has a positive effect on increasing the working range of the ASA. The reason for this phenomenon is that the enhancement of $d_{l}$ expands the angle range of impedance matching, thus, the AAR is also enlarged. In Fig.7, as $d_{A}$ differs from $0.46 d_{l}$ to $0.54 d_{l}$ with an interval of $0.02 d_{1}$, the AAR hardly changes, always being $-36.5^{\circ} \sim 36.5^{\circ}$, but the absorptance has been obviously influenced. 
Only when $d_{A}$ is equal to $0.5 d_{l}$, can it show excellent absorption performance. Under other conditions, the absorptance will be significantly lower than 0.9 , threatening the filtering performance of the ASA. Therefore, only when the precondition of $d_{A}=0.5 d_{l}$ is satisfied, the effect of the anti-reflection structure can be exerted to the best situation and the optimized absorption effect can be produced.

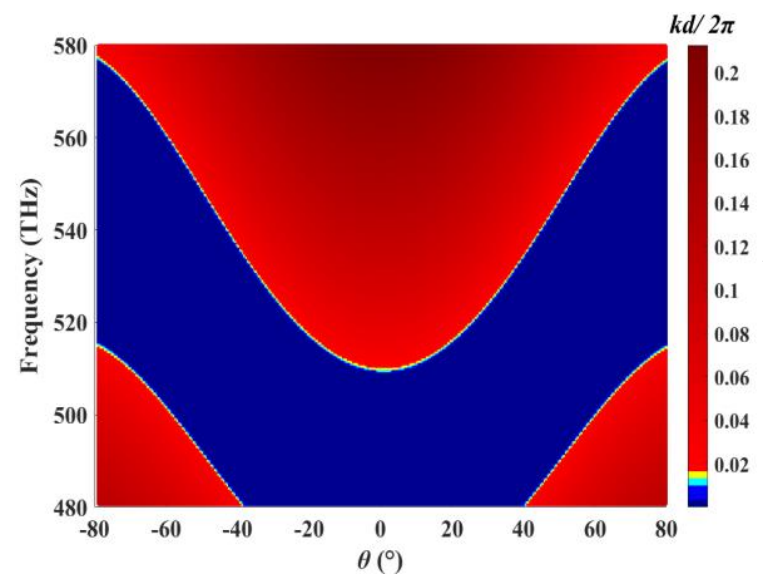

Fig.5. The graph of the relationship between the dispersion and $\theta$ under frequency conditions. $k$ represents wave vector, and $d=d_{l}+d_{B}$.

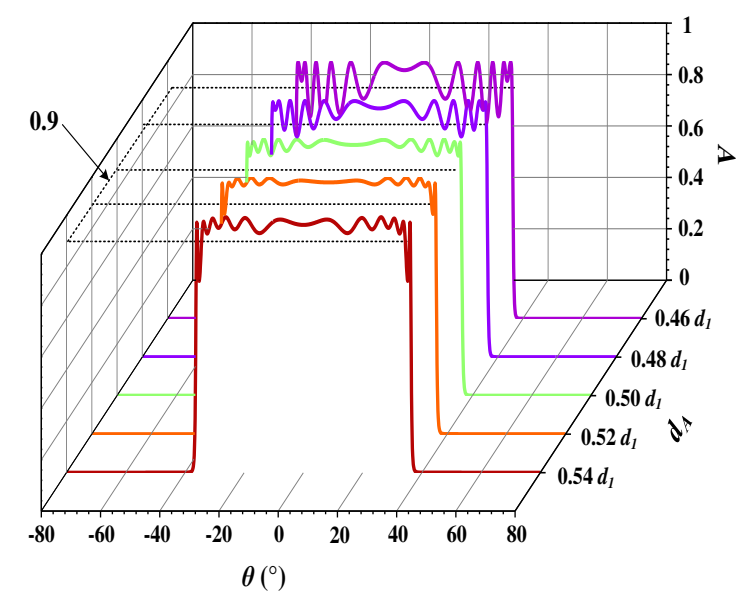

Fig.7. The effect of the relationship between $d_{A}$ and $d_{l}$ on the AAR.

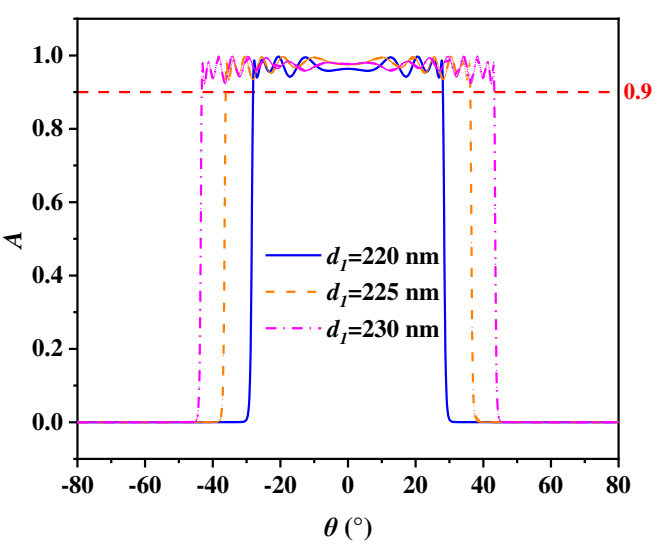

Fig.6. The influence of the thickness of $d_{l}$ on the AAR.

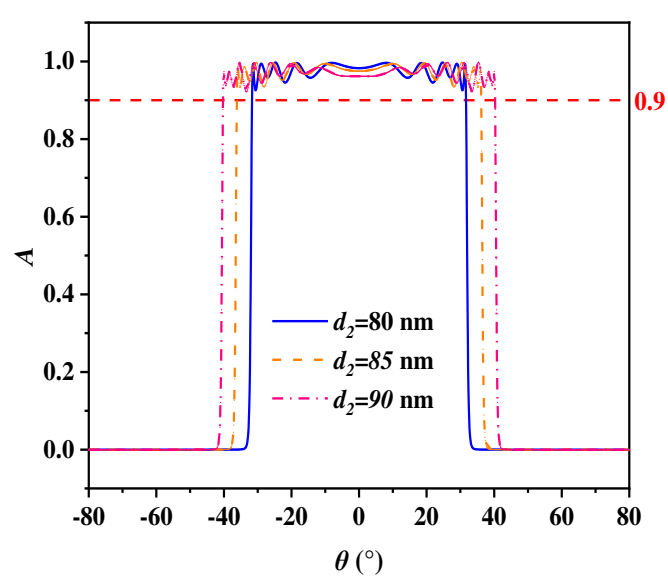

Fig.8. The influence of the thickness of $d_{2}$ on the AAR.

In Fig. 8 , in case that $d_{2}$ is $80 \mathrm{~nm}$, its AAR is $-32^{\circ} \sim 32^{\circ}$; if $d_{2}$ is changed to $85 \mathrm{~nm}$, the AAR becomes $-36.5^{\circ} \sim 36.5^{\circ}$; suppose $d_{2}=90 \mathrm{~nm}$, the AAR will be changed to $-40.6^{\circ} \sim 40.6^{\circ}$. The increase of $d_{2}$ will respectively expand the AAR by $9^{\circ}$ and $8.2^{\circ}$, but the intensity of the increase is not as strong as that caused by $d_{l}$. Similarly, during the change of $d_{2}$, the absorptance is always higher than 0.9 and the $B$ value is maintained at 0.95 . Hence, a proper extension in $d_{2}$ can also promote the expansion of the working range of the ASA. This can be attributed to that the augment in $d_{2}$ makes a wider range of impedance matching well, so the AAR is also expanded. In Fig.9, when $d_{B}$ is $0.91 d_{2}, 0.93 d_{2}$, $0.95 d_{2}, 0.97 d_{2}$, or $0.99 d_{2}$, the impact on the ASA has been investigated, but only when $d_{B}=0.95 d_{2}$, the performance of the ASA is outstanding. Under other conditions, the impedance matching will be misaligned to a certain extent, so that the absorptance will decrease, and it will not completely reach more than 0.9 . Consequently, the relationship between $d_{B}$ and $d_{2}$ is fixed after all and cannot be changed at will. 


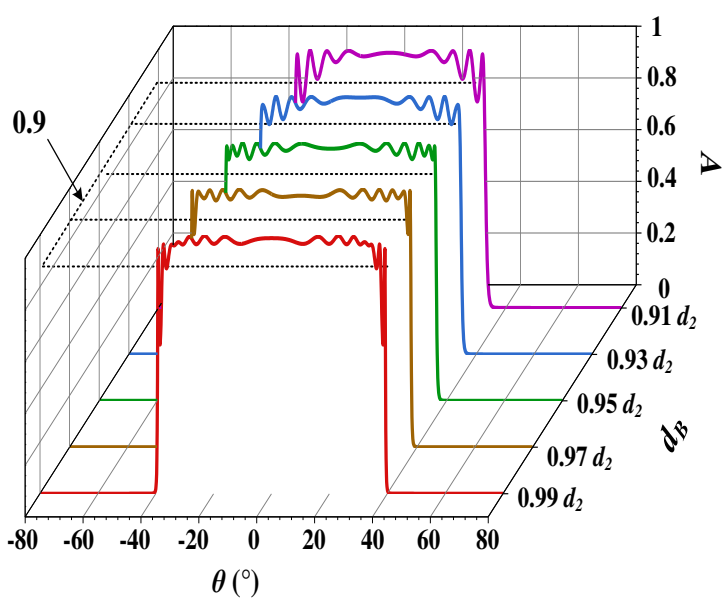

Fig.9. The influence of the relation between $d_{B}$ and $d_{2}$ on AAR.
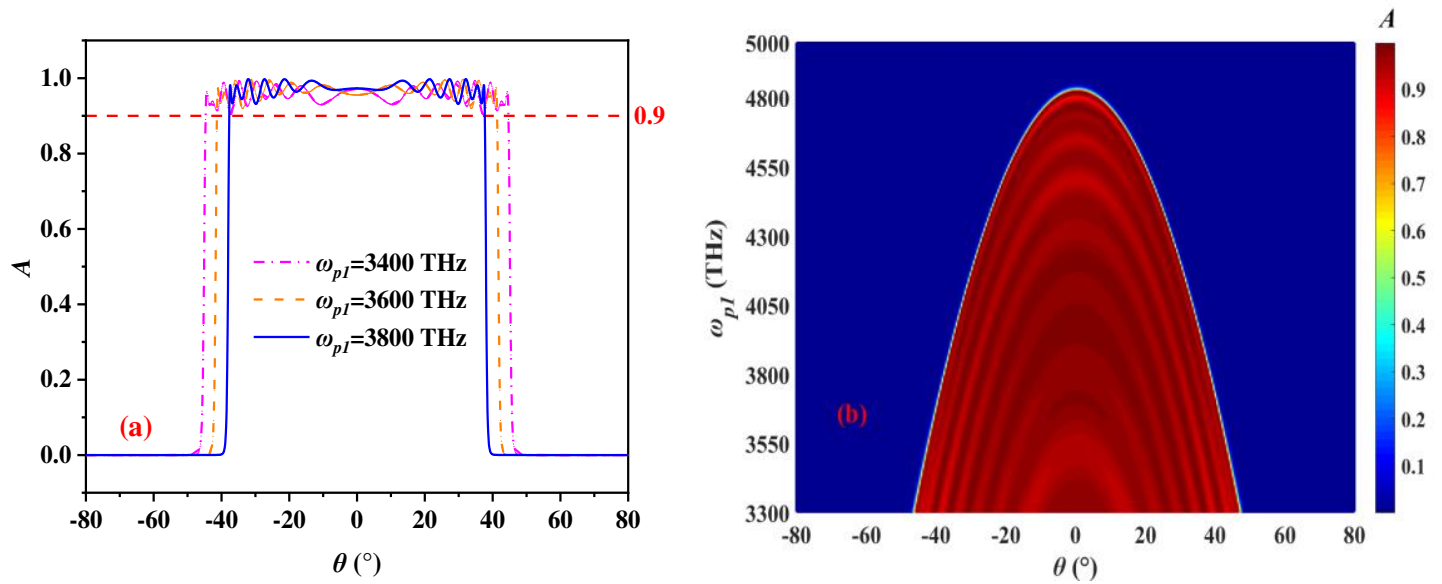

Fig.10. (a) The map of the AAR when $\omega_{p l}=3400 \mathrm{THz}, 3600 \mathrm{THz}$ or $3800 \mathrm{THz}$. (b) The effect of continuously changing $\omega_{p l}$ on the AAR.

In Fig.10(a), the augment of $\omega_{p l}$ has a reduced effect on the AAR. In the case of $\omega_{p l}=3400 \mathrm{THz}$, the AAR is $-45.1^{\circ} \sim 45.1^{\circ}$. If $\omega_{p 1}$ becomes $3600 \mathrm{THz}$, the AAR will decrease to $-41.7^{\circ} \sim 41.7^{\circ}$. The AAR is reduced by $6.8^{\circ}$. When $\omega_{p 1}$ increases to $3800 \mathrm{THz}$, the AAR will change to $-38^{\circ} \sim 38^{\circ}$, and decrease again by $7.4^{\circ}$. It is worth mentioning that during the change of $\omega_{p 1}$, only the AAR has been evidently reduced, the absorptance is still above 0.9 , and $B$ is always overtopped. Since the three-dimensional top view can more clearly see the adjustment of the continuous $\omega_{p 1}$ to the AAR, Fig.10(b) is shown. Because the amplification of $\omega_{p 1}$ lessens the range of impedance matching and also broadens the photonic band gap in the dispersion curve, the AAR meeting the conditions will also decline. On condition that the $\omega_{p l}$ is greater than $4800 \mathrm{THz}$, the AAR disappears to 0 . In this process, the absorptance will always maintain a high value, as revealed in the red area in the figure, and the edge of the red area always keeps a smooth state, so $B$ will also be maintained productively. 

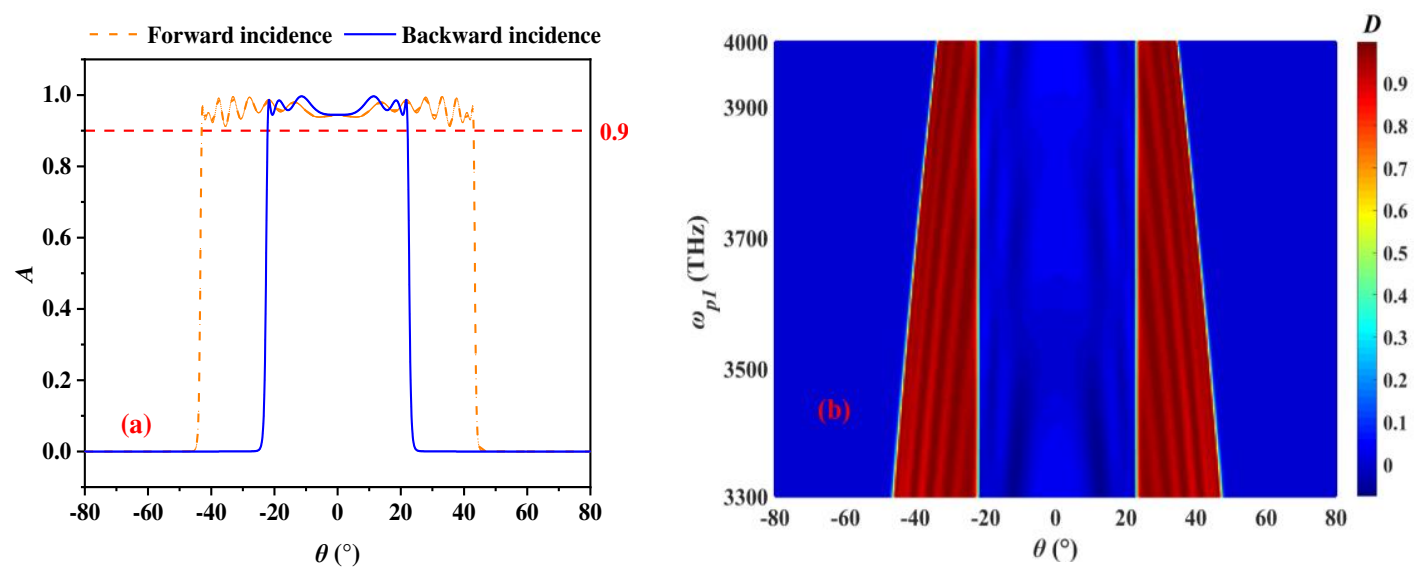

Fig.11. (a) The NP of the ASA. (b) The effect of $\omega_{p 1}$ on the NP.

In Fig.1, the thickness of the 72nd layer of ITO is very large, and $\omega_{p 3}=5000 \mathrm{THz}$ is also very large. This arrangement completely absorbs the incident EM wave, making it unable to propagate into the following structure. Based on this principle, we adopt the same arrangement structure on both sides of the 72nd layer, but the parameter settings are inconsistent, thus realizing the phenomenon of nonreciprocity. In order to highlight the degree of nonreciprocity, $D$ is defined to describe. $D=|F-H|$, where $F$ and $H$ refer to the absorptance of the forward and backward incidences, respectively. The value of $D$ ranges from $0 \sim 1$. The larger the value of $D$, the stronger the NP, and the more obvious the difference between forward and backward incidences. Here we change $\omega_{p 1}$ to $3500 \mathrm{THz}$, which can better emphasize the NP. As revealed in Fig.11(a), the AAR is $-43.5^{\circ} \sim 43.5^{\circ}$ for the forward incidence and $-20.4^{\circ} \sim 20.4^{\circ}$ for the backward incidence. They all maintain a high absorptance of 0.9 and a good $B$ value of 0.95. The forward and backward incident EM waves are clearly separated in the range of $-43.5^{\circ} \sim-20.4^{\circ}$ and $20.4^{\circ} \sim 43.5^{\circ}$. Ulteriorly, this NP is tunable. According to the previous analysis, $d_{l}, d_{2}$, and $\omega_{p l}$ can be utilized to adjust for this phenomenon. But considering the flexibility of actual operation, $\omega_{p l}$ is chosen. In Fig. 11(b), with the increase of $\omega_{p l}$, the area of high $D$ value (the red area in the figure) is obviously decreasing. In the process of the adjustment, the $B$ value is always in a good state, which can be seen from the smooth edge of the red area.

\section{Conclusion}

In this paper, we present a one-dimensional periodic multilayer structure containing ITO thin films to analyze the ASA and its NP. The tuning effects of $d_{1}, d_{2}$, and $\omega_{p l}$ on the ASA and NP are also discussed. The results show that the two conditions $d_{A}=0.5 d_{1}$ and $d_{B}=0.95 d_{2}$ are the prerequisites for ensuring the excellent performance of the ASA. The dispersion theory and impedance matching theory are introduced to explain the reasons. Ingenious structural design is also an important reason to ensure a high absorptance and high $B$ value. In previous studies on the ASFDs, the research on the ASAs based on one-dimensional layered structures like ours is rare, let alone exploring its NP. Consequently, we hope that our proposed method can provide new design ideas for manufacturing new ASAs.

\section{Acknowledgments}

This work was supported by the Open Research Program in China's State Key Laboratory of Millimeter Waves (Grant No. K201927).

\section{References}


[1] B.Guo, M.X.Gao, L.Peng, X.Cai, "Optical Properties of One-Dimensional Multi-Layered Structures Containing Plasma Materials", Applied Mechanics and Materials , 110(7):585-591(2012).

[2] F. Romeo and R. Citro, "Spin-torque generation by dc or ac voltages in quasi-one-dimensional magnetic layered structures", Physical Review B, 81(4):045307(2010).

[3] M. F.O. Hameed, M. Y. Azab, A. M. Heikal, and S. M. ElHefnawy, S. S. A. Obayya, "Highly sensitive plasmonic photonic crystal temperature sensor fifilled with liquid crystal”, IEEE Photonics Technology Letters, 28(3):59-62(2015).

[4] A. Anopchenko, A. Occhicone, R. Rizzo, and A. Sinibaldi, "Effect of thickness disorder on the performance of photonic crystal surface wave sensors", Optics Express, 24(7):7728-7742(2016).

[5] P. B. Bing, J. L. Sui, G. F. Wu , X.Y. Guo, Z.Y. Li, L. Tan, and J. Q. Yao, "Analysis of Dual-Channel Simultaneous Detection of Photonic Crystal Fiber Sensors", Plasmonics, 2020, doi: 10.1007/s11468-020-01131-9.

[6] H. N`emec and P. Kužel, "Highly tunable photonic crystal filter for the terahertz range", Optics Letters, 30(5): 549(2005).

[7] J. He, L. T. Song, H. L. Wang, Y. A. Han, and T. L, "Angular tuning of defect modes spectra for one dimensional photonic crystal filters with a liquid crystal layer", Optoelectronics Letters, 7(6): 0437(2011).

[8] H. Iizuka, N. Engheta, and S. Sugiura, "Extremely small wavevector regime in a one-dimensional photonic crystal heterostructure for angular transmission filtering”, Optics Letters, 41(16):3829(2016).

[9] J. F. Zhu, C. W. Li, J. Y. Ou, and Q. H. Liu, "Perfect light absorption in graphene by two unpatterned dielectric layers and potential applications", Carbon, 142: 430(2019).

[10] C. W. Li, J. L. Qiu, J. Y. Ou, Q. H. Liu, and J. F. Zhu, "High-sensitivity refractive index sensors using coherent perfect absorption on graphene in the Vis-NIR region," ACS Applied Nano Materials, 2:3231-3237(2019).

[11] S. K. Patel, S. Charola, R. Jadeja,T. K. Nguyen, and V. Dhasarathan, "Wideband graphene-based near-infrared solar absorber using C-shaped rectangular sawtooth metasurface", Physica E: Low-dimensional Systems and Nanostructures, https://doi.org/10.1016/j.physe.2020.114493.

[12] H. P. Zheng, C. H. Tian, and Y. F. Li, “ Tunable frequency and angular photonic crystal filter”, Proceedings of SPIE, 6787:678117(2016).

[13] H. Tanaka, I. Takai, H. Fujikawa, and H. Iizuka, “ Nearly Polarization-Independent Angular Filters Consisting of One-Dimensional Photonic Crystals Realized in the Visible Region", Journal of Lightwave Techonology, 36(12):2517(2018).

[14] G. Liang, P. Han, and H. Wang, "Narrow frequency and sharp angular defect mode in one-dimensional photonic crystals from a photonic het erostructure," Optics Letters, 29(2):192-194(2004).

[15] R. E. Hamam, I. Celanovic, and M. Soljacic, "Angular photonic band gap," Physics Review A, 83:035806(2011).

[16] A. Alu, G. D. Aguanno, N. Mattiucci, and M. J. Bloemer, "Plasmonic Brewster angle: Broadband extraordinary transmission through optical gratings," Physics Review Letters,106:123902(2011).

[17] Y. Shen, D. Ye, I. Celanovic, S. G. Johnson, J. D. Joannopoulos, and M. Soljacic, "Optical broadband angular selectivity," Science, 343:1499-1501(2014).

[18] D. C. Serrano, J. S. G. Diaz, D. L. Sounas, Y. Hadad, A. A. Melcon, and A. Alú, "Nonreciprocal graphene devices and antennas based on spatiotemporal modulation", IEEE Antennas Wireless Propagation Letters, 15:1529-1532(2016).

[19] C. Fang, Y. Liu, G. Han, Y. Shao, J. Zhang, and Y. Hao, "Localized plasmon resonances for black phosphorus bowtie nanoantennas at terahertz frequencies”, Optics Express, 26:27683-27693(2018). 
[20] C. M. Chen, J. Xu, and Y. Yao, "SIW resonator humidity sensor based on layered black phosphorus", Electronics Letters, 53(4):249-251(2017).

[21] J. Yoon, M. Zhou, M. A. Badsha, T. Y. Kim, Y. C. Jun, and K. H. Chang, "Broadband epsilon-near-zero perfect absorption in the near-infrared", Science Reports, 5:12788(2015).

[22] G. Palikaras, H. Hu, J. Atkinson, R. Fedosejevs, R. Starkobowes, S. Pramanik, T. Kallos, W. Newman, and Z. Jacob, "Optical characterization of epsilon-near-zero, epsilon-near-pole, and hyperbolic response in nanowire metamaterials", JOSA B, 32(10): 2074-2080(2015).

[23] K. T. Young, B. M. Alamgir, Y. Junho, L. S. Young, J. Y. Chul, and H. C. Kwon, "General strategy for broadband coherent perfect absorption and multi-wavelength all-optical switching based on epsilon-nearzero multilayer films", Science Reports, 6:22941(2016).

[24] K. Zhou, Q. Cheng, J. Song, L. Lu, Z. H. Jia, and J. W. Li, "Broadband perfect infrared absorption by tuning epsilon-near-zero and epsilon-near-pole resonances of multilayer ITO nanowires", Applied Optics, 57(1):102(2018).

[25] L. M. Qi, Z. Q. Yang, F. Lan, X. Gao, and Z.J.Shi, "Properties of obliquely incident electromagnetic wave in one-dimensional magnetized plasma photonic crystals", Physics of Plasmas , 2010, dol: 10.1063/1.3360296

[26] S. J. Guo and C. X. Hu, "Ultra-wide unidirectional infrared absorber based on 1D gyromagnetic photonic crystals concatenated with general Fibonacci quasi-periodic structure in transverse magnetizatio", Journal of Optics, 22:105101(2020).

[27] Q. Bai, B. Z. Zhang, and J. P. Duan, “ Design of W band Rectangular Waveguide Filter”, Application of Electronic Technique, 44:66(2018).

[28] T. W. Chang, C. H. Huang, D. J. Hou, C. J. Wu, and D. X. Chen, "Analysis of unidirectional absorption in a defective superconducting photonic crystal," IEEE Photonics Journal, 9(4):1-9(2017). 